\title{
Blow-up solution and stability to an inverse problem for a pseudo-parabolic equation
}

M Yaman ${ }^{*}$

*Correspondence:

myaman@sakarya.edu.tr

Department of Mathematics,

Sakarya University, Sakarya, Turkey

\section{Abstract \\ We consider a two-fold problem for an inverse problem of pseudo-parabolic equations with a nonlinear term. Sufficient conditions for a blow-up solution are derived and a stability result is established.}

Keywords: blow-up; inverse problem; pseudo-parabolic equation; stability

\section{Introduction}

Let us consider the following inverse problem for a pseudo-parabolic equation:

$$
\begin{aligned}
& u_{t}-a \Delta u_{t}-\Delta u+\sum_{i=1}^{n} b_{i} u_{x_{i}}-|u|^{p} u=f(t) g(x), \quad x \in \Omega, t>0, \\
& u(x, t)=0, \quad x \in \partial \Omega, t>0, \\
& u(x, 0)=u_{0}(x), \quad x \in \Omega, \\
& \int_{\Omega} u(x, t) g(x) d x=1, \quad t>0,
\end{aligned}
$$

where $\Omega \subset R^{n}$ is a bounded domain with a sufficiently smooth boundary $\partial \Omega, p$ and $a$ are positive constants, $g(x)$ and $b_{i}(x)$ are given functions satisfying

$$
\omega \in H^{2}(\Omega) \cap H_{0}^{1}(\Omega) \cap L^{p+2}(\Omega), \quad \int_{\Omega} \omega(\omega-\Delta \omega) d x=1,
$$

with weight function $g(x)=\omega-a \Delta \omega$, and a constant

$$
B_{0}=\max _{x \in \Omega}\left(\sum_{i=1}^{n} b_{i}^{2}(x)\right)^{1 / 2}, \quad x \in \Omega, b_{i} \in C(\bar{\Omega}) .
$$

The inverse problem consists of finding a pair of functions $(u(x, t), f(t))$ satisfying (1)-(4) when

$$
u_{0} \in H_{0}^{1}(\Omega) \cap L^{p+2}(\Omega) \quad \text { and } \quad \int_{\Omega} u_{0}(\omega-\Delta \omega) d x=1 .
$$

Additional information about the solution to the inverse problem is given in the form of the integral overdetermination condition (4). From the physical point of view, this condition may be interpreted as measurements of the temperature $u(x, t)$ by a device averaging over the domain $\Omega$ [1].

@ 2012 Yaman; licensee Springer. This is an Open Access article distributed under the terms of the Creative Commons Attribution License (http://creativecommons.org/licenses/by/2.0), which permits unrestricted use, distribution, and reproduction in any medium, provided the original work is properly cited. 
This type of equations arises from a variety of mathematical models in engineering and physical sciences; for example, inverse scattering problems in quantum physics, an inverse problem of interest in geophysics [2].

Existence and uniqueness of solutions to an inverse problem for parabolic and pseudoparabolic equations are studied in [3-6]. Stability of solutions is investigated by several authors [1, 7]; but less is known about blow-up solutions. Eden and Kalantarov [8] studied the same problem without a strong damping term $-\Delta u_{t}$. Meyvaci [9] established a blowup result for the pseudo-parabolic equation $u_{t}-\Delta u_{t}-\Delta u+u_{x_{1}} u^{p}=|u|^{2 m} u$, where $p \geq 1$ is a given integer and $m \geq 1$ is a number.

Here, we used the following notations:

$$
\|u\|=\|u\|_{L_{2}(\Omega)}, \quad(u, v)=\int_{\Omega} u v d x, \quad\|u\|_{p}=\|u\|_{L^{p}(\Omega)}
$$

are the arithmetic-geometric inequality and Young's inequality for $a, b>0$ respectively;

$$
a b \leq \frac{\varepsilon}{2} a^{2}+\frac{1}{2 \varepsilon} b^{2}, \quad a b \leq \beta a^{p}+C(p, \beta) b^{q},
$$

with $1 / p+1 / q=1, C(p, \beta)=\frac{1}{q(\beta p)^{q / p}}$ and the Poincare-Friedrich inequality

$$
\lambda_{1}\|u\|^{2} \leq\|\nabla u\|^{2}
$$

where $\lambda_{1}$ is the first eigenvalue of the eigenvalue problem

$$
-\Delta u=\lambda u, \quad x \in \Omega ; \quad u=0, \quad x \in \partial \Omega .
$$

Multiplying both sides of (1) by $\omega$ and integrating the resulting equation over $\Omega$ lead to the following relation:

$$
f(t)=-(u, \Delta \omega)+\left(\omega, \sum_{i=1}^{n} b_{i} u_{x_{i}}\right)-\left(\omega,|u|^{p} u\right),
$$

where conditions (2), (3) and (A1) are used. Substituting (7) into (1), problem (1)-(3) yields a direct problem given by [4].

\section{Blow-up result}

Firstly, let us note the following lemma known as 'generalized concavity lemma' or 'Ladyzhenskaya-Kalantarov lemma'. It is an important tool to obtain the blow-up solutions to parabolic- and hyperbolic-type equations.

Lemma 1 Let $\alpha>0, C_{1}, C_{2} \geq 0$ and $C_{1}+C_{2}>0$. Suppose that a positive, twice differentiable function $F(t)$ satisfies the inequality

$$
F(t) F^{\prime \prime}(t)-(1+\alpha)\left(F^{\prime}(t)\right)^{2} \geq-2 C_{1} F(t) F^{\prime}(t)-C_{2} F^{2}(t), \quad \forall t \geq 0 .
$$

If

$$
F(0)>0 \text { and } F^{\prime}(0)+\gamma_{2} \alpha^{-1} F(0)>0,
$$


then $F(t)$ goes to infinity as

$$
t \rightarrow t_{1} \leq t_{2}=\frac{1}{2 \sqrt{C_{1}^{2}+C_{2}}} \ln \frac{\gamma_{1} F(0)+\alpha F^{\prime}(0)}{\gamma_{2} F(0)+\alpha F^{\prime}(0)} .
$$

Here, $\gamma_{1}=-C_{1}+\sqrt{C_{1}^{2}+\alpha C_{2}}$ and $\gamma_{2}=-C_{1}-\sqrt{C_{1}^{2}+\alpha C_{2}}$.

Proof See [10].

Theorem 1 Assume that (A1)-(A3) are satisfied and suppose that the initial condition $u_{0}$ satisfies the following condition:

$$
\begin{aligned}
\frac{2(2 p+3)}{p+2}\left\|u_{0}\right\|_{p+2}^{p+2}> & 2 \sqrt{\frac{4}{a^{2}}+\frac{2(p+1)^{2}}{a p^{2}}\left(2 B_{0}^{2}+K_{0}^{2}\right)}\left(\left\|u_{0}\right\|^{2}+a\left\|\nabla u_{0}\right\|^{2}\right) \\
& -\left(1+B_{0}^{2}\right)\left\|u_{0}\right\|^{2}-D_{1},
\end{aligned}
$$

where $K_{0}>0$ and $D_{1}=\|\Delta \omega\|^{2}+B_{0}^{2}\|\omega\|^{2}+\frac{2}{p+2}\|\omega\|_{p+2}^{p+2}$. Then the solution of the problem (1)-(4) with the weight function $g(x)=(\omega-a \Delta \omega)(x)$ blows up in a finite time.

Proof Multiplying (1) by $u$ and integrating over $\Omega$ give

$$
\frac{1}{2} \frac{d}{d t}\left(\|u\|^{2}+a\|\nabla u\|^{2}\right)+\|\nabla u\|^{2}+\left(u, \sum_{i=1}^{n} b_{i} u_{x_{i}}\right)-\|u\|_{p+2}^{p+2}=f(t) .
$$

Also, multiplying (1) by $u_{t}$ and integrating over $\Omega$, we obtain

$$
\left\|u_{t}\right\|^{2}+a\left\|\nabla u_{t}\right\|^{2}=-\frac{1}{2} \frac{d}{d t}\|\nabla u\|^{2}-\left(u_{t}, \sum_{i=1}^{n} b_{i} u_{x_{i}}\right)+\frac{1}{p+2} \frac{d}{d t}\|u\|_{p+2}^{p+2} .
$$

Now, let us consider the following function:

$$
F(t)=\|u\|^{2}+a\|\nabla u\|^{2}+D_{0}
$$

where $D_{0}$ is a nonnegative parameter to be chosen later. It is clear that

$$
F^{\prime}(t)=2\left(u, u_{t}\right)+2 a\left(\nabla u, \nabla u_{t}\right) .
$$

Using the Cauchy-Schwarz inequality, we have

$$
\left(F^{\prime}(t)\right)^{2} \leq 4 F(t)\left(\left\|u_{t}\right\|^{2}+a\left\|\nabla u_{t}\right\|^{2}\right) .
$$

Substituting (11) into (12), we obtain

$$
\begin{aligned}
\left\|u_{t}\right\|^{2}+a\left\|\nabla u_{t}\right\|^{2}= & \frac{1}{2(p+2)} F^{\prime \prime}(t)-\frac{p}{2(p+2)} \frac{d}{d t}\|\nabla u\|^{2} \\
& -\left(u_{t}, \sum_{i=1}^{n} b_{i} u_{x_{i}}\right)+\frac{1}{p+2} \frac{d}{d t}\left(u, \sum_{i=1}^{n} b_{i} u_{x_{i}}\right)-\frac{1}{p+2} \frac{d}{d t} f(t) .
\end{aligned}
$$


We take the derivative of (7) with respect to $t$

$$
\frac{d}{d t} f(t)=-\left(u_{t}, \Delta \omega\right)+\left(\omega, \sum_{i=1}^{n} b_{i} u_{t x_{i}}\right)-(p+1)\left(\omega, u^{p} u_{t}\right)
$$

Rewrite (16) in view of (17)

$$
\begin{aligned}
\left\|u_{t}\right\|^{2}+a\left\|\nabla u_{t}\right\|^{2}= & \frac{1}{2(p+2)} F^{\prime \prime}(t)-\frac{p}{2(p+2)} \frac{d}{d t}\|\nabla u\|^{2}-\frac{p+1}{p+2}\left(u_{t}, \sum_{i=1}^{n} b_{i} u_{x_{i}}\right) \\
& +\frac{1}{p+2}\left(u, \sum_{i=1}^{n} b_{i} u_{t x_{i}}\right)+\frac{1}{p+2}\left(u_{t}, \Delta \omega\right) \\
& -\frac{1}{p+2}\left(\omega, \sum_{i=1}^{n} b_{i} u_{t x_{i}}\right)+\frac{p+1}{p+2}\left(\omega,|u|^{p} u_{t}\right) .
\end{aligned}
$$

After applying the arithmetic-geometric inequality to estimate the terms on the right-hand side of (18), we obtain

$$
\begin{aligned}
& \frac{d}{d t}\|\nabla u\|^{2} \leq 2 \int_{\Omega} \nabla u \cdot \nabla u_{t} d x, \\
& 2\left|\int_{\Omega} \nabla u \cdot \nabla u_{t} d x\right| \leq \frac{4}{a}\|\nabla u\|^{2}+\frac{a}{4}\left\|\nabla u_{t}\right\|^{2}, \\
& \left(u_{t}, \sum_{i=1}^{n} b_{i} u_{x_{i}}\right) \leq \frac{p}{8(p+1)}\left\|u_{t}\right\|^{2}+\frac{2 B_{0}^{2}(p+1)}{p}\|\nabla u\|^{2}, \\
& \left(u, \sum_{i=1}^{n} b_{i} u_{t x_{i}}\right) \leq \frac{2 B_{0}^{2}}{a p}\|u\|^{2}+\frac{a p}{8}\left\|\nabla u_{t}\right\|^{2}, \\
& \left|\left(u_{t}, \Delta \omega\right)\right| \leq \frac{p}{8}\left\|u_{t}\right\|^{2}+\frac{2}{p}\|\Delta \omega\|^{2}, \\
& \left|\left(\omega, \sum_{i=1}^{n} b_{i} u_{t x_{i}}\right)\right| \leq \frac{B_{0}^{2}}{a p}\|\omega\|^{2}+\frac{a p}{4}\left\|\nabla u_{t}\right\|^{2} .
\end{aligned}
$$

For $q=\frac{2 n}{n-2}, n \geq 3$, the following inequality is satisfied for some $K_{1}>0$ :

$$
\left(\int_{\Omega}|u|^{q} d x\right)^{1 / q} \leq K_{1}\left(\int_{\Omega}|\nabla u|^{2} d x\right)^{1 / 2}
$$

We apply the Hölder inequality, with $q_{1}=n, q_{2}=2, q_{3}=\frac{2 n}{n-2}$, to the last term in (18),

$$
\left.\left|\int_{\Omega} \omega\right| u\right|^{p} u_{t} d x \mid \leq\left(\int_{\Omega}|\omega|^{n} d x\right)^{1 / n}\left(\int_{\Omega}\left|u_{t}\right|^{2} d x\right)^{1 / 2}\left(\int_{\Omega}|u|^{\frac{2 n}{n-2}} d x\right)^{\frac{n-2}{2 n}}
$$

It follows from (24) and (25) with $\|\omega\|_{n} \leq K_{2}$

$$
K_{0}\left\|u_{t}\right\|\|\nabla u\| \leq \frac{p}{4(p+1)}\left\|u_{t}\right\|^{2}+\frac{p+1}{p} K_{0}^{2}\|\nabla u\|^{2},
$$


where $K_{0}=K_{1} K_{2}$. Substituting the estimates (19)-(23) and (26) into (18), we write

$$
\begin{aligned}
\frac{p+4}{2(p+2)}\left(\left\|u_{t}\right\|^{2}+a\left\|\nabla u_{t}\right\|^{2}\right) \leq & \frac{1}{2(p+2)} F^{\prime \prime}(t)+\frac{2 B_{0}^{2}}{a p(p+2)}\|u\|^{2} \\
& +\frac{2 p a^{-1}+p^{-1}(p+1)^{2}\left(2 B_{0}^{2}+K_{0}^{2}\right)}{a(p+2)} a\|\nabla u\|^{2} \\
& +\frac{1}{p(p+2)}\left(2\|\Delta \omega\|^{2}+\frac{B_{0}^{2}}{a}\|\omega\|^{2}\right) .
\end{aligned}
$$

Since coefficients of the term $a\|\nabla u\|^{2}$ are greater than those of $\|u\|^{2}$ on the right-hand side of (27), multiplying both sides of $(27)$ by $2(p+2)$, we get

$$
\begin{aligned}
& (p+4)\left(\left\|u_{t}\right\|^{2}+a\left\|\nabla u_{t}\right\|^{2}\right) \\
& \leq F^{\prime \prime}(t)+\left(\frac{4 p}{a^{2}}+\frac{2(p+1)^{2}}{a p}\left(2 B_{0}^{2}+K_{0}^{2}\right)\right)\left(\|u\|^{2}+a\|\nabla u\|^{2}\right)+D_{2}
\end{aligned}
$$

where $D_{2}=\frac{4}{p}\|\Delta \omega\|^{2}+\frac{2 B_{0}^{2}}{a p}\|\omega\|^{2}$. From (15) and (28), we have

$$
\left(1+\frac{p}{4}\right) F^{-1}(t)\left(F^{\prime}(t)\right)^{2} \leq F^{\prime \prime}(t)+\beta F(t)+\left(D_{2}-\beta D_{0}\right)
$$

where $\beta=\frac{4 p}{a^{2}}+\frac{2(p+1)^{2}}{a p}\left(2 B_{0}^{2}+K_{0}^{2}\right)$. We choose $D_{0}=\beta^{-1} D_{2}$ in the last inequality and multiply both sides of (29) by $F(t)$, which gives

$$
F(t) F^{\prime \prime}(t)-\left(1+\frac{p}{4}\right)\left(F^{\prime}(t)\right)^{2} \geq-\beta(F(t))^{2}
$$

So, inequality (8) is satisfied with $\alpha=\frac{p}{4}>0, C_{1}=0, C_{2}=\beta>0$. Thus, the desired result is obtained by applying Lemma 1 .

\section{Stability of problem}

In this part, we consider the following inverse source problem:

$$
\begin{aligned}
& u_{t}-\Delta u_{t}-\Delta u-\sum_{i=1}^{n} b_{i} u_{x_{i}}+|u|^{p} u=f(t) \omega(x), \quad x \in \Omega, t>0, \\
& u(x, t)=0, \quad x \in \partial \Omega, t>0, \\
& u(x, 0)=u_{0}(x), \quad x \in \Omega, \\
& \int_{\Omega} u(x, t) \omega(x) d x=\varphi(t),
\end{aligned}
$$

where $\Omega \subset R^{n}$ is a bounded domain with a sufficiently smooth boundary $\partial \Omega$ and $\omega, u_{0}$ and $\varphi(t)$ are given functions, $p>0$. Assume that $\omega$ satisfies the conditions

$$
\int_{\Omega} \omega^{2} d x=1, \quad \omega \in H_{0}^{1}(\Omega) \cap L^{p+2}(\Omega)
$$


and $u_{0}$ satisfies

$$
u_{0} \in H_{0}^{1}(\Omega) \cap L^{p+2}(\Omega) \text { and } \int_{\Omega} u_{0}(x) \omega(x) d x=\varphi(0) .
$$

Theorem 2 Suppose that the conditions (A5) and (A6) are satisfied and assume that $\varphi$ and $\varphi^{\prime}$ are continuous functions defined on $[0, \infty)$ which tend to zero as $t \rightarrow \infty$. Then

$$
\lim _{t \rightarrow \infty}\left(\|\nabla u\|^{2}+\|u\|_{p+2}^{p+2}\right)=0
$$

with a constant $B_{0}<\frac{2\left(\sqrt{1+\lambda_{1}}-1\right)}{\sqrt{\lambda_{1}}}$, where $\lambda_{1}$ is constant in (6).

Proof We multiply (31) by $\omega$, integrate over $\Omega$ and use (34) to obtain

$$
f(t)=\varphi^{\prime}(t)+\left(\nabla \omega, \nabla u_{t}\right)+(\nabla \omega, \nabla u)-\left(\omega, \sum_{i=1}^{n} b_{i} u_{x_{i}}\right)+\left(\omega,|u|^{p} u\right) .
$$

Inserting (35) into (31), we obtain

$$
\begin{aligned}
u_{t} & -\Delta u_{t}-\Delta u-\sum_{i=1}^{n} b_{i} u_{x_{i}}+|u|^{p} u \\
& =\left(\varphi^{\prime}(t)+\left(\nabla \omega, \nabla u_{t}\right)+(\nabla \omega, \nabla u)-\left(\omega, \sum_{i=1}^{n} b_{i} u_{x_{i}}\right)+\left(\omega,|u|^{p} u\right)\right) \omega(x) .
\end{aligned}
$$

Now, let us multiply (36) by $u+u_{t}$ and integrate over $\Omega$ :

$$
\begin{aligned}
\frac{d}{d t}[ & \left.\frac{1}{2}\|u\|^{2}+\|\nabla u\|^{2}+\frac{1}{p+2}\|u\|_{p+2}^{p+2}\right]+\|\nabla u\|^{2}+\|u\|_{p+2}^{p+2}+\left\|u_{t}\right\|^{2}+\left\|\nabla u_{t}\right\|^{2} \\
= & \left(\varphi^{\prime}(t)+\varphi(t)\right)\left[\varphi^{\prime}(t)+\left(\nabla \omega, \nabla u_{t}\right)+(\nabla \omega, \nabla u)-\left(\omega, \sum_{i=1}^{n} b_{i} u_{x_{i}}\right)+\left(\omega,|u|^{p} u\right)\right] \\
& +\left(u+u_{t}, \sum_{i=1}^{n} b_{i} u_{x_{i}}\right) .
\end{aligned}
$$

Using Cauchy, Poincare and Young inequalities on the right-hand side of (37), we have

$$
\begin{aligned}
& \left|\left(u, \sum_{i=1}^{n} b_{i} u_{x_{i}}\right)\right| \leq \frac{B_{0}}{\sqrt{\lambda_{1}}}\|\nabla u\|^{2}, \\
& \left|\left(u_{t}, \sum_{i=1}^{n} b_{i} u_{x_{i}}\right)\right| \leq\left\|u_{t}\right\|^{2}+\frac{B_{0}^{2}}{4}\|\nabla u\|^{2}, \\
& \left|\left(\nabla \omega, \nabla u_{t}\right)\left(\varphi^{\prime}+\varphi\right)\right| \leq\left\|\nabla u_{t}\right\|^{2}+\frac{1}{2}\|\nabla \omega\|^{2}\left(\left|\varphi^{\prime}\right|^{2}+|\varphi|^{2}\right), \\
& \left|(\nabla \omega, \nabla u)\left(\varphi^{\prime}+\varphi\right)\right| \leq \frac{\varepsilon}{2}\|\nabla u\|^{2}+\frac{1}{\varepsilon}\|\nabla \omega\|^{2}\left(\left|\varphi^{\prime}\right|^{2}+|\varphi|^{2}\right), \\
& \left|\left(\omega, \sum_{i=1}^{n} b_{i} u_{x_{i}}\right)\left(\varphi^{\prime}+\varphi\right)\right| \leq \frac{\varepsilon}{2}\|\nabla u\|^{2}+\frac{B_{0}^{2}}{\varepsilon}\|\omega\|^{2}\left(\left|\varphi^{\prime}\right|^{2}+|\varphi|^{2}\right), \\
& \left|\left(\omega,|u|^{p} u\right)\left(\varphi^{\prime}+\varphi\right)\right| \leq \varepsilon\|u\|_{p+2}^{p+2}+C(\varepsilon, p)\|\omega\|_{p+2}^{p+2}\left(\left|\varphi^{\prime}\right|^{p+2}+|\varphi|^{p+2}\right) .
\end{aligned}
$$


Rewriting (37) with estimates (38)-(43), we obtain the following inequality:

$$
\begin{aligned}
\frac{d}{d t} & {\left[\frac{1}{2}\|u\|^{2}+\|\nabla u\|^{2}+\frac{1}{p+2}\|u\|_{p+2}^{p+2}\right] } \\
& +\left(1-\varepsilon-\frac{B_{0}\left(4+B_{0} \sqrt{\lambda_{1}}\right)}{4 \sqrt{\lambda_{1}}}\right)\|\nabla u\|^{2}+(1-\varepsilon)\|u\|_{p+2}^{p+2} \leq D(t),
\end{aligned}
$$

where

$$
\begin{aligned}
D(t)= & \left(\left|\varphi^{\prime}\right|^{2}+|\varphi|^{2}\right)\left(\frac{1}{2}\|\nabla \omega\|^{2}+\frac{1}{\varepsilon}\|\nabla \omega\|^{2}+\frac{B_{0}^{2}}{\varepsilon}\|\omega\|^{2}\right) \\
& +\left(\varphi^{\prime}(t)\right)^{2}+\left|\varphi^{\prime}(t) \varphi(t)\right|+C(\varepsilon, p)\|\omega\|_{p+2}^{p+2}\left(\left|\varphi^{\prime}\right|^{p+2}+|\varphi|^{p+2}\right) .
\end{aligned}
$$

We choose $\varepsilon_{0}>0$ such that $\varepsilon_{0} \leq \varepsilon<1-\frac{B_{0}\left(4+B_{0} \sqrt{\lambda_{1}}\right)}{4 \sqrt{\lambda_{1}}}$ and take

$$
K_{3}=\min \left\{\frac{2}{3}\left(1-\varepsilon_{0}-\frac{B_{0}\left(4+B_{0} \sqrt{\lambda_{1}}\right)}{4 \sqrt{\lambda_{1}}}\right), 1-\varepsilon_{0}\right\} .
$$

So, (44) follows

$$
\frac{d}{d t}\left[\frac{1}{2}\|u\|^{2}+\|\nabla u\|^{2}+\frac{1}{p+2}\|u\|_{p+2}^{p+2}\right]+K_{3}\left(\frac{3}{2}\|\nabla u\|^{2}+\|u\|_{p+2}^{p+2}\right) \leq D(t) .
$$

The last term on the left-hand side of (45) can be written

$$
\begin{aligned}
\frac{3}{2}\|\nabla u\|^{2}+\|u\|_{p+2}^{p+2} & \geq \frac{\lambda_{1}}{2}\|u\|^{2}+\|\nabla u\|^{2}+\|u\|_{p+2}^{p+2} \\
& \geq K_{4}\left(\frac{1}{2}\|u\|^{2}+\|\nabla u\|^{2}+\frac{1}{p+2}\|u\|_{p+2}^{p+2}\right),
\end{aligned}
$$

where $K_{4}=\min \left(\lambda_{1}, 1\right)$. It follows from (45) and (46)

$$
\frac{d}{d t} \eta(t)+K_{5} \eta(t) \leq D(t)
$$

Here, $K_{5}=K_{3} K_{4}$ and $\eta(t)=\frac{1}{2}\|u\|^{2}+\|\nabla u\|^{2}+\frac{1}{p+2}\|u\|_{p+2}^{p+2}$. After solving first-order differential inequality (47), it follows that

$$
\|\nabla u\|^{2}+\|u\|_{p+2}^{p+2} \rightarrow 0 \quad \text { as } t \rightarrow \infty
$$

\section{Competing interests}

The author declares that they have no competing interests.

Received: 20 February 2012 Accepted: 14 November 2012 Published: 28 November 2012

\section{References}

1. Vasin, $V$, Kamynin, L: On the asymptotic behaviour of solutions to inverse problems for parabolic equations. Sib. Math. J. 38, 647-662 (1997)

2. Ramm, AG: Inverse Problems, Mathematical and Analytical Techniques with Applications to Engineering. Springer, New York (2005) 
3. Kamynin, VL, Franchini, E: An inverse problem for a higher order parabolic equation. Math. Notes 64(5), 590-599 (1998)

4. Prilepko, Al, Orlowskii, DG, Vasin, IA: Methods for Solving Inverse Problems in Mathematical Physics. Dekker, New York (2000)

5. Riganti, R, Savateev, E: Solution of an inverse problem for the nonlinear heat equation. Commun. Partial Differ. Equ. 19, 1611-1628 (1994)

6. Tkachenko, DS: On an inverse problem for a parabolic equation. Math. Notes 75(5), 729-743 (2004)

7. Guvenilir, AF, Kalantarov, VK: The asymptotic behaviour of solutions to an inverse problem for differential operator equations. Math. Comput. Model. 37, 907-914 (2003)

8. Eden, A, Kalantarov, VK: On global behaviour of solutions to an inverse problem for nonlinear parabolic equations. J. Math. Anal. Appl. 307, 120-133 (2005)

9. Meyvaci, M: Blow-up of solutions of pseudo-parabolic equations. J. Math. Anal. Appl. 352, 629-633 (2009)

10. Kalantarov, VK, Ladyzhenskaya, OA: Formation of collapses in quasilinear equations of parabolic and hyperbolic types. Zap. Nauč. Semin. LOMI 69, 77-102 (1977)

doi:10.1186/1029-242X-2012-274

Cite this article as: Yaman: Blow-up solution and stability to an inverse problem for a pseudo-parabolic equation. Journal of Inequalities and Applications 2012 2012:274.

\section{Submit your manuscript to a SpringerOpen ${ }^{\circ}$ journal and benefit from:}

- Convenient online submission

- Rigorous peer review

- Immediate publication on acceptance

- Open access: articles freely available online

- High visibility within the field

- Retaining the copyright to your article 